\title{
BMJ Open Assessing the impact of simplified HCV care on linkage to care amongst high- risk patients at primary healthcare clinics in Malaysia: a prospective observational study
}

To cite: Markby J, Shilton S, Sem X, et al. Assessing the impact of simplified HCV care on linkage to care amongst high-risk patients at primary healthcare clinics in Malaysia: a prospective observational study. BMJ Open 2021;11:e055142. doi:10.1136/ bmjopen-2021-055142

- Prepublication history and additional supplemental material for this paper are available online. To view these files, please visit the journal online (http://dx.doi.org/10.1136/ bmjopen-2021-055142).

$\mathrm{JM}$ and SS are joint first authors.

Received 06 July 2021 Accepted 29 November 2021

Check for updates

(c) Author(s) (or their employer(s)) 2021. Re-use permitted under CC BY-NC. No commercial re-use. See rights and permissions. Published by BMJ.

For numbered affiliations see end of article.

Correspondence to

Sonjelle Shilton

sonjelle.shilton@finddx.org

\section{ABSTRACT}

Introduction To achieve the elimination of hepatitis C virus (HCV), substantial scale-up in access to testing and treatment is needed. This will require innovation and simplification of the care pathway, through decentralisation of testing and treatment to primary care settings and task-shifting to non-specialists. The objective of this study was to evaluate the feasibility and effectiveness of decentralisation of HCV testing and treatment using rapid diagnostic tests (RDTs) in primary healthcare clinics (PHCs) among high-risk populations, with referral of seropositive patients for confirmatory viral load testing and treatment. Methods This observational study was conducted between December 2018 and October 2019 at 25 PHCs in three regions in Malaysia. Each PHC was linked to one or more hospitals, for referral of seropositive participants for confirmatory testing and pretreatment evaluation. Treatment was provided in PHCs for non-cirrhotic patients and at hospitals for cirrhotic patients.

Results During the study period, a total of 15366 adults were screened at the 25 PHCs, using RDTs for HCV antibodies. Of the 2020 (13.2\%) HCV antibody-positive participants, 1481/2020 (73.3\%) had a confirmatory viral load test, 1241/1481 (83.8\%) were HCV RNA-positive, 991/1241 (79.9\%) completed pretreatment assessment, 632/991 (63.8\%) initiated treatment, 518/632 (82.0\%) completed treatment, 352/518 (68.0\%) were eligible for a sustained virological response (SVR) cure assessment, 209/352 (59.4\%) had an SVR cure assessment, and SVR was achieved in 202/209 (96.7\%) patients. A significantly higher proportion of patients referred to PHCs initiated treatment compared with those who had treatment initiated at hospitals $(71.0 \%$ vs $48.8 \%, p<0.001)$.

Conclusions This study demonstrated the effectiveness and feasibility of a simplified decentralised HCV testing and treatment model in primary healthcare settings, targeting

\section{Strengths and limitations of this study}

A strength of this study is the ability to assess associations of hepatitis C virus (HCV) positivity and demographic factors and risk factors.

- A strength of this study is the comparison of retention in care cascade between participants initiated in treatment at district hospital compared with primary health clinic.

- A strength of this study is that it is a pragmatic study of feasibility of decentralised HCV care integrated into existing health system in Malaysia which led to national scale up of aspects of the study model.

- A limitation of this study is the rates of treatment initiation were not as high as targeted, impacted in large part by COVID-19 related disruptions.

high-risk groups in Malaysia. There were good outcomes across most steps of the cascade of care when treatment was provided at PHCs compared with hospitals.

\section{INTRODUCTION}

Hepatitis $\mathrm{C}$ virus (HCV) is a major cause of chronic liver disease globally, with an estimated 58 million individuals chronically infected and 290000 HCV-related deaths each year. ${ }^{1-3}$ In 2016, the WHO launched the Global Health Sector Strategy on Hepatitis 2016-2021, ${ }^{4}$ with the goal of eliminating viral hepatitis as a public health threat by 2030 . However, as of 2019, just $21 \%$ of individuals with HCV infection worldwide had been 
tested and approximately one-quarter of diagnosed individuals had been treated. ${ }^{3}$

The global response for the elimination of HCV infection have been transformed by recent advances in treatment and diagnostics, as well as reductions in costs. These advances include direct-acting antiviral (DAA) therapy and the availability of point-of-care serological and nucleic acid testing for HCV. The development of evidence-based WHO guidelines on who and how to test has provided further support for the scale-up of testing and treatment. ${ }^{25}$

Malaysia is an upper middle-income country of more than 32 million people and an estimated HCV seroprevalence in the general population between $0.3 \%$ and 2.5\%. ${ }^{67}$ People who inject drugs (PWID) represent just $0.24 \%$ (75 000) of the adult population; however, they have an HCV prevalence of $67.5 \%-89.9 \% .^{7}$ Other key populations in Malaysia at higher risk of HCV include 77 903 people living with HIV (PLHIV) $(0.24 \%$ of the population), ${ }^{8} 221698$ men who have sex with men (MSM) $(0.69 \%), 22000$ female sex workers (FSWs) $(0.069 \%)$ and 15000 transgender sex workers (TGSWs) $(0.047 \%) .{ }^{9}$

In 2017, it was estimated that only 6.1\% (23 258) of people infected with $\mathrm{HCV}$ were diagnosed. ${ }^{10-12} \mathrm{~A}$ likely cause of this low rate of diagnosis was the highly complex and centralised testing model used. Prior to the commencement of this project in Malaysia, to screen for HCV antibodies, staff at primary healthcare clinics (PHCs) send samples to a central laboratory leading to long turnaround times and loss to follow-up. An overall goal of the national programme is to expand HCV services to the 1027 PHCs nationally. ${ }^{13} 14$

The objective of this study was to demonstrate the feasibility and effectiveness of decentralisation of HCV testing using rapid diagnostic tests (RDTs) at PHCs among highrisk populations, with referral of seropositive patients for confirmatory viral load testing and treatment. Effectiveness was evaluated through retention across the HCV care cascade. A further objective was to derive lessons learnt and to inform scale-up of HCV national and regional strategies.

\section{METHODS}

\section{Study design and settings}

This was an observational, prospective cohort study (figure 1 and online supplemental figure 1), with enrolment conducted between December 2018 and October 2019 in three regions of Malaysia: (a) the state of Kedah, (b) the state of Kelantan and (c) the region of Kuala Lumpur/Putrajaya/state of Selangor. This observational study was designed to evolve with the national HCV programme and therefore included several protocol changes during the study duration outlined below. This enabled the possibility to carry out several subanalyses within the study that were not initially part of the study outcomes. This study was also designed to feed eligible RNA positive participants into a clinical trial entitled
'Open label phase II/III, multicentre trial to assess the efficacy, safety, tolerance and pharmacokinetics of sofosbuvir plus ravidasvir in HCV (+/-HIV) chronically infected adults with no or compensated cirrhosis in Thailand and Malaysia' (Malaysian Medical Research Ethics Committee, approval number NMRR-16-747-29183, coordinated by DND $i$, hereafter called the DND $i$ trial). ${ }^{15} 16$

\section{Study outcomes}

The outcomes of the study were the proportion of patients with a positive anti-HCV RDT who have a confirmatory HCV RNA test done, the proportion of patients with a positive HCV RNA test result who initiate hepatitis $\mathrm{C}$ treatment. Additional outcomes included; the proportion of patients who tested positive when screened for anti-HCV using RDT, the time required to progress from anti-HCV screening to in the HCV care cascade and the primary cost and resource use of the HCV care cascade services including screening, confirmatory test, pretreatment assessment, monitoring and treatment.

\section{Site selection}

Twenty-five PHCs were selected for enrolment and screening of participants (online supplemental figure 1). Site feasibility assessments were conducted for 31 PHCs recommended by the Ministry of Health $(\mathrm{MOH})$, based on the existence of a methadone maintenance therapy programme, presence of a family medicine specialist, sufficient staffing and proximity to the catchment area of five selected hospitals $(<100 \mathrm{~km})$ (online supplemental table 1). Sites were selected using a points-based system coupled with a laboratory assessment. Each PHC was linked to one or more hospitals for referral of seropositive participants (Hospital Sultanah Bahiyah in the state of Kedah, Hospital Raja Perempuan Zainab II in the state of Kelantan, and Hospital Selayang, Hospital Ampang, and Hospital Sungai Buloh in the region of Kuala Lumpur/ Putrajaya/state of Selangor). The median distance from selected PHCs to the selected hospitals was $29.6 \mathrm{~km}$.

\section{Study participants}

Adult participants were enrolled consecutively at the 25 PHCs, based on routine clinical indications for an $\mathrm{HCV}$ test as per the Malaysian national guidelines ${ }^{17}$ and according to one of the following HCV risk factors (obtained either based on routine triage and/or clinical indications as per national guidelines, self-reported or obtained from medical records): a history of invasive medical procedures (eg, surgery, biopsy, endoscopy, solid organ donation); long-term haemodialysis; received blood/blood products/clotting factor concentrates/ organ transplant prior to 1994; a needle-stick injury or mucosal exposure to HCV-infected blood; chronic liver disease and/or hepatitis; tattoos; body piercing; born to an HCV-infected mother; has an HCV-infected partner; is an MSM; is transgender; is an SW; was previously in prison; is HIV-positive; injects drugs; uses illicit intranasal drugs; has any other or undisclosed risk of HCV. Patients 


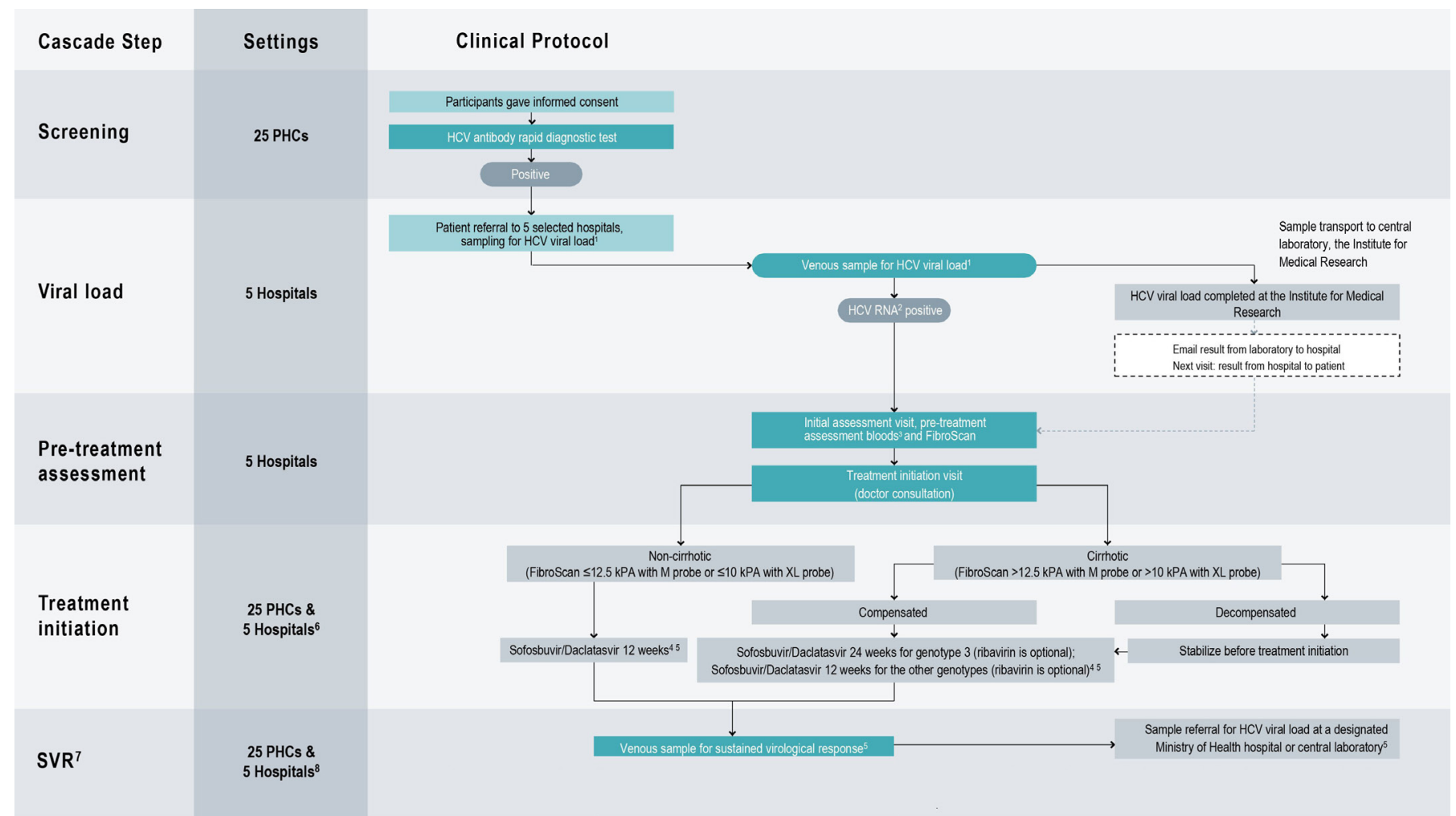

Figure 1 Hepatitis $\mathrm{C}$ virus $(\mathrm{HCV})$ care pathway at primary healthcare clinics (PHCs) and hospitals. ${ }^{1}$ Pre-enrolment requirements by Drugs for Neglected Diseases initiative (DNDi) trials for HCV viral load at the Institute for Medical Research. ${ }^{2}$ Ribonucleic acid. ${ }^{3}$ Pretreatment assessment bloods: complete blood count, liver function tests-aspartate aminotransferase (AST) and alanine aminotransferase (ALT) and the subsequent list is needed for DNDi trial assessment: genotyping, alkaline phosphatase, direct and indirect bilirubin, creatinine. ${ }^{4}$ Ministry of Health Malaysia, Management of Chronic Hepatitis C in Adults - Clinical Practice Guidelines 2019. MOH/P/PAK/433.19(GU)-e. ${ }^{5}$ Does not include the 67 patients enrolled in DNDi trial. ${ }^{6}$ Refer to figure 3 for flowchart of patients that initiated treatment at PHCs and hospitals. ${ }^{7}$ Sustained virological response (done in 12-week to 24week window after end of treatment). ${ }^{8} \mathrm{As}$ the study period ended on 31 October 2020, some patients had completed treatment by this date but were not yet eligible for SVR testing, therefore data were not collected for these patients for the purposes of the study. These patients were however offered SVR testing through the Ministry of Health programme.

already diagnosed as HCV RNA-positive or already initiated on treatment for the management of HCV infection were excluded from the study.

\section{Study procedures}

HCV screening

Eligible study participants were enrolled at PHCs and following pretest counselling, offered anti-HCV screening using finger-stick capillary or venous blood and tested with an SD Bioline HCV RDT (Standard Diagnostic, Korea). If the result was positive, participants were referred to one of the five selected hospitals for confirmatory testing by appointment (2-4 weeks after screening).

\section{Confirmatory testing and pre-treatment evaluations}

At the selected hospital, a $10 \mathrm{~mL}$ venous blood sample was drawn into EDTA-containing tubes and plasma was prepared within 72 hours, then referred to a reference laboratory in Kuala Lumpur (Institute of Medical Research, IMR) for HCV RNA testing using the Roche cobas $4800 \mathrm{HCV}$ assay. The HCV RNA results were returned to the hospital and, at a subsequent patient visit. A second venous blood sample $(5 \mathrm{~mL})$ was obtained from patients who were HCV RNA-positive, for pretreatment evaluations. This sample was tested at the hospital laboratory and included a full blood evaluation and liver function tests measuring alanine aminotransferase, aspartate aminotransferase, bilirubin (direct/indirect), alkaline phosphatase and serum creatinine. At the selected hospitals, patients also received a FibroScan to assess the presence of cirrhosis (cirrhosis: $>12.5 \mathrm{kPA}$ with an $\mathrm{M}$ probe or $>10 \mathrm{kPA}$ with an XL probe, absence of cirrhosis: $\leq 12.5 \mathrm{kPA}$ with an M probe or $\leq 10 \mathrm{kPA}$ with an XL probe). Venous blood $(10 \mathrm{~mL})$ was obtained for genotyping which was carried out at IMR using the Roche cobas $4800 \mathrm{HCV}$ GT assay.

Treatment and evaluation of cure

Following a clinical evaluation, participants were referred for enrolment into the $\mathrm{DND} i$ trial. ${ }^{15} 16$ If patients were eligible and gave their written informed consent to take part in the DND $i$ trial, they were also initiated on treatment and managed as per the DND $i$ trial. Patients who were not eligible or who did not give consent to participate in the DND $i$ trial were referred to the standard of 
care, the MOH national programme under which. Participants were treated for 12 weeks or 24 weeks depending on cirrhotic status.

At 12-24 weeks after the end of treatment, patients were requested to return to the treatment centre for a final venous blood sample to be collected $(5 \mathrm{~mL})$ for sustained virological response (SVR) HCV viral load testing, with plasma referral for testing at a designated $\mathrm{MOH}$ hospital or central laboratory. Patients with treatment failure were referred for further management by the gastroenterology or hepatology specialist, in accordance to the consensus of the 2019 National Clinical Practice Guidelines Development Group. ${ }^{18}$ Treatment outcomes for the 67 RNApositive DND $i$ trial enrolees were embargoed until published separately and therefore have been excluded from the treatment outcomes of this publication. The screened population however could not be identified for exclusion from the final analysis.

\section{Protocol changes during the study}

At the commencement of the study, HCV treatment in Malaysia was delivered through the $\mathrm{MOH}$ national programme in hospitals. However, during the study duration (quarter 3, 2019), the national guidelines were changed to recommend treatment of non-cirrhotic patients (including those coinfected with HIV) at PHCs under the care of family medicine specialists. This enabled a subanalysis of treatment outcomes for patients that received treatment at hospitals versus those that received treatment at PHCs. Cirrhotic patients continued to be treated at hospitals; however, a subgroup of compensated cirrhotic patients $(n=59)$ were treated (using sofosbuvir/ daclatasavir without ribavirin ${ }^{18} 19$ at six PHCs (in the state of Kedah).

\section{Patient and public involvement}

Public involvement, via civil society groups, included sharing the protocol design for review and input during the conception phase as well as active participation of civil society groups in results dissemination activities.

\section{Data collection and analysis}

Data were collected from primary source documents (screening registers, patient medical records, laboratory registers and laboratory reports) using paper case report forms (pCRFs) at PHCs by PHC study staff. These pCRFs were then manually transcribed into electronic case report forms (eCRFs) by research assistants using OpenClinica enterprise version 3.14 open-source software. At the hospitals, data were directly collected using eCRFs.

To ensure data quality, regular site monitoring visits were carried out (one visit per month), with every CRF checked for completeness and general errors. In addition, both manual and automated data cleaning were carried out on completed database exports.

Data were analysed using $\mathrm{R}$ V.3.6.1 to provide descriptive and inferential statistics. Characteristics of HCV antibody-positive and HCV antibody-negative individuals were summarised according to demographic, clinical, laboratory and treatment categories, with median and IQRs for quantitative data and frequencies and percentages for qualitative data. Associations between demographic characteristics and the frequency of HCV-positive patients were assessed using simple and multiple logistic regression (S/MLR). With MLR, all other demographic factors were accounted for by including them in the model as covariates. Variables examined included age, sex, ethnicity, antenatal status, reported risk factors and the total number of confirmed risk factors for each patient. Resulting $\mathrm{p}$ values were adjusted for multiple hypotheses using the BenjaminiHochberg method.

Outcomes across the cascade of care were reported as numbers and percentages for each step for total populations and separately for cirrhotic/non-cirrhotic, hospital/ PHC and key population (PWID/non-PWID, PLHIV/ non-PLHIV) subgroups. Similarly, the times between HCV care cascade steps were reported as median and IQR values. Subgroup outputs at each step of the care cascade and turnaround time analyses were compared using Pearson's $\chi^{2}$ test. Multiple hypothesis adjustment for subgroup comparison was performed using the Bonferroni correction. ${ }^{20}$ Associations between SVR output and demographic characteristics of treated patients were assessed using Pearson's $\chi^{2}$ test.

\section{Assessment of costs}

Estimates of the costs associated with testing were collected from the study sites. An ingredients-based approach was used to estimate the average cost per person of an antibody test and an RNA test. Unit costs included the costs of diagnostics tests and other consumables used; staff time, recorded as minutes of healthcare worker, administrative staff and laboratory technician time (with costs assigned by multiplying average minutes spent by salary); and overheads, including a proportion of the costs of utilities, phones, computers and other equipment (with costs assigned by dividing the annual or one-off cost of each item by the estimated number of appointments in a year or its estimated lifetime). Estimates of costs associated with treatment and auxiliary tests, such as liver function tests, were provided by the MOH. To assess the relative costeffectiveness of the testing and care pathways, we used a state-transition model, MATCH (Markov-based Analyses of Treatments for Chronic Hepatitis C), which simulates HCV disease progression. Natural history outcomes from this model have been validated previously. ${ }^{21-23}$ We adapted this model to simulate the epidemiology of HCV in Malaysia (MATCH-Malaysia) and extended the model to evaluate the cost-effectiveness of the three different care pathways: the total cohort, the treatment pathway at the PHCs and the treatment pathway at the hospitals. The model was developed following the principles of economic analyses with respect to viral hepatitis recommended by WHO. ${ }^{24}$ 


\section{RESULTS}

\section{Study population characteristics}

A total of 15366 adults from 25 PHCs in three regions (3933 $(25.6 \%)$ in the state of Kedah, 3717 (24.2\%) in the state of Kelantan and $7716(50.2 \%)$ in the region of Kuala Lumpur/Putrajaya/state of Selangor) were screened using HCV antibody RDTs between December 2018 and October 2019 (table 1). The median (IQR) age was $38(30-50)$ years, $9122(59.4 \%)$ were male and $12315(80.1 \%)$ were of Malay ethnicity. In terms of self-reported risk factors for HCV exposure, a significant proportion (38.2\%) did not disclose any specific risk factors. The most common risk factors reported were body piercings $(21.1 \%)$, a history of invasive medical procedures $(18.2 \%)$, injection drug use $(13.0 \%)$, intranasal illicit drug use $(14.7 \%)$ and previous imprisonment $(12.4 \%)$.

\section{Factors associated with HCV antibody positivity}

Overall, 2087 were HCV RDT positive (13.6\%), and this was similar across the three regions $(15.6 \%, 12.2 \%$ and $13.3 \%$ HCV RDT positivity in the state of Kedah, the state of Kelantan and the region of Kuala Lumpur/Putrajaya/ state of Selangor, respectively) (table 1). RDT-positive participants were on average 7 years older than RDTnegative patients (44 (39-52) vs 37 (29-49) years, respectively, $\mathrm{p}<0.001)$ and were more likely to be male $(21.6 \%$ vs $1.8 \%, \mathrm{p}<0.001)$. In the MLR analysis, after adjustment for age and sex, the reported risk factors for exposure that were most strongly associated with RDT positivity, compared with their absence, were injecting drug use $(\mathrm{OR}=28.3,95 \% \mathrm{CI} 24.3$ to 33.0, $\mathrm{p}<0.001)$, history of haemodialysis $(\mathrm{OR}=5.2,95 \%$ CI 2.7 to $10.0, \mathrm{p}<0.001)$ and blood transfusion ( $\mathrm{OR}=4.9,95 \% \mathrm{CI} 3.3$ to $7.4, \mathrm{p}<0.001$ ), followed by history of illicit intranasal drug use $(\mathrm{OR}=2.0$, $95 \% \mathrm{CI} 1.8$ to $2.3, \mathrm{p}<0.001)$, history of incarceration $(\mathrm{OR}=2.4,95 \%$ CI 2.0 to $2.8, \mathrm{p}<0.001)$ and HIV infection $(\mathrm{OR}=2.3,95 \% \mathrm{CI} 1.8$ to $2.9, \mathrm{p}<0.001)$. A history of chronic liver disease was also strongly associated with HCV antibody positivity ( $\mathrm{OR}=6.0,95 \%$ CI 4.7 to $7.7, \mathrm{p}<0.001) .4473$ (29.1\%) participants had 2-4 risk factors and 208 (1.4\%) Participants had more than four risk factors, having two of more risk factors was strongly associated with HCV antibody positivity, compared with participants with one risk factor $(\mathrm{p}<0.001)$.

\section{Outcomes across the HCV care cascade}

Overall, of the $2020 \mathrm{HCV}$ antibody positive participants, $1481 / 2020(73.3 \%)$ had a confirmatory viral load test performed, 1241/1481 (83.8\%) were HCV RNA positive, 991/1241 $(79.9 \%)$ had pretreatment assessments completed, 632/991 (63.8\%) initiated treatment, $518 / 632(82 \%)$ completed treatment, 352/518 (68.0\%) were eligible for SVR cure assessment at the time of study completion, 209/352 (59.4\%) had an SVR cure assessment and 202/209 (96.7\%) patients achieved SVR (figure 2).
Among those who initiated treatment, the majority $(452 / 632,71.5 \%)$ were non-cirrhotic and received a 12-week regimen of sofosbuvir/daclatasvir (figure 3), while 180/632, 28.5\% were cirrhotic (of whom, 21/632 (3.3\%) were decompensated) (data not shown) and received 12-week or 24-week (depending on genotype) treatment regimen of sofosbuvir/daclatasvir.

Of the 991 patients who were HCV RNA positive and completed the pretreatment assessments, $660(66.6 \%)$ were non-cirrhotic and $331(33.4 \%)$ were cirrhotic. Of the 660 non-cirrhotic patients, $596(90.3 \%)$ were referred to a PHC for treatment and $64(9.7 \%)$ were referred to a hospital for treatment. Of those referred, 416/596 $(69.8 \%)$ initiated treatment at a PHC and 36/64 (56.3\%) initiated treatment at a hospital, with both groups receiving a 12-week treatment regimen of sofosbuvir/daclatasvir. During the study period, a total of 208/660 (31.5\%) noncirrhotic patients were not initiated on treatment. Of the 331 cirrhotic patients (of which $23(7.4 \%)$ had decompensated cirrhosis), $73(22.1 \%)$ were referred to a PHC for treatment and $258(77.9 \%)$ were referred to a hospital for treatment. Of those referred, 59/73 $(80.8 \%)$ initiated treatment at a PHC and 121/258 (46.9\%) initiated treatment at a hospital, with both groups receiving a 12-week or 24-week (depending on genotype) treatment regimen of sofosbuvir/daclatasvir. During the study period, a total of $151 / 331(45.6 \%)$ cirrhotic patients were not initiated on treatment. Of the $359 / 1241(36.2 \%)$ patients that did not initiate treatment: 197/359 (54.9\%) were LTFU; reason was not available for $81 / 359(22.6 \%)$; 55/359 (15.2\%) had other underlying comorbidities and paused HCV treatment; 10/359 (2.8\%) were incarcerated; 8/359 (2.2\%) patients died (6: unknown reasons, 2: hepatoma/ advanced retroviral disease); 6/359 (1.7\%) patients did not want to start treatment and 2/359 (0.6\%) patients were ineligible for free treatment through the $\mathrm{MOH}$.

Seven serious adverse events were reported during the study period, none were assessed to be caused by the study procedures/interventions. Two participants were hospitalised due to injuries associated with accidents, five participants died (one accident, one stroke, one heart failure, one pneumonia and one chronic liver disease (death was prior to HCV confirmatory testing)).

Cascade outcomes for non-cirrhotic and cirrhotic patients referred to PHCs or hospitals for treatment were similar except for the following: the treatment initiation rate among cirrhotic patients referred to PHCs was significantly higher $(80.8 \%)$ than those referred to hospitals (46.9\%, $\mathrm{p}<0.001)$; the treatment completion rate among non-cirrhotic patients referred to PHCs was also significantly higher $(94.0 \%)$ than those referred to hospitals $(69.4 \%, \mathrm{p}<0.001)$.

Overall, there were $47 / 632$ (7.4\%, data not shown) participants lost to follow-up after treatment initiation, of whom similar proportions of participants were cirrhotic $(25 / 47(53.2 \%))$ versus non-cirrhotic $(22 / 47(46.8 \%))$. Of those lost to follow-up, $20(42.6 \%)$ initiated treatment at a PHC and $27(57.4 \%)$ initiated treatment at a hospital. 


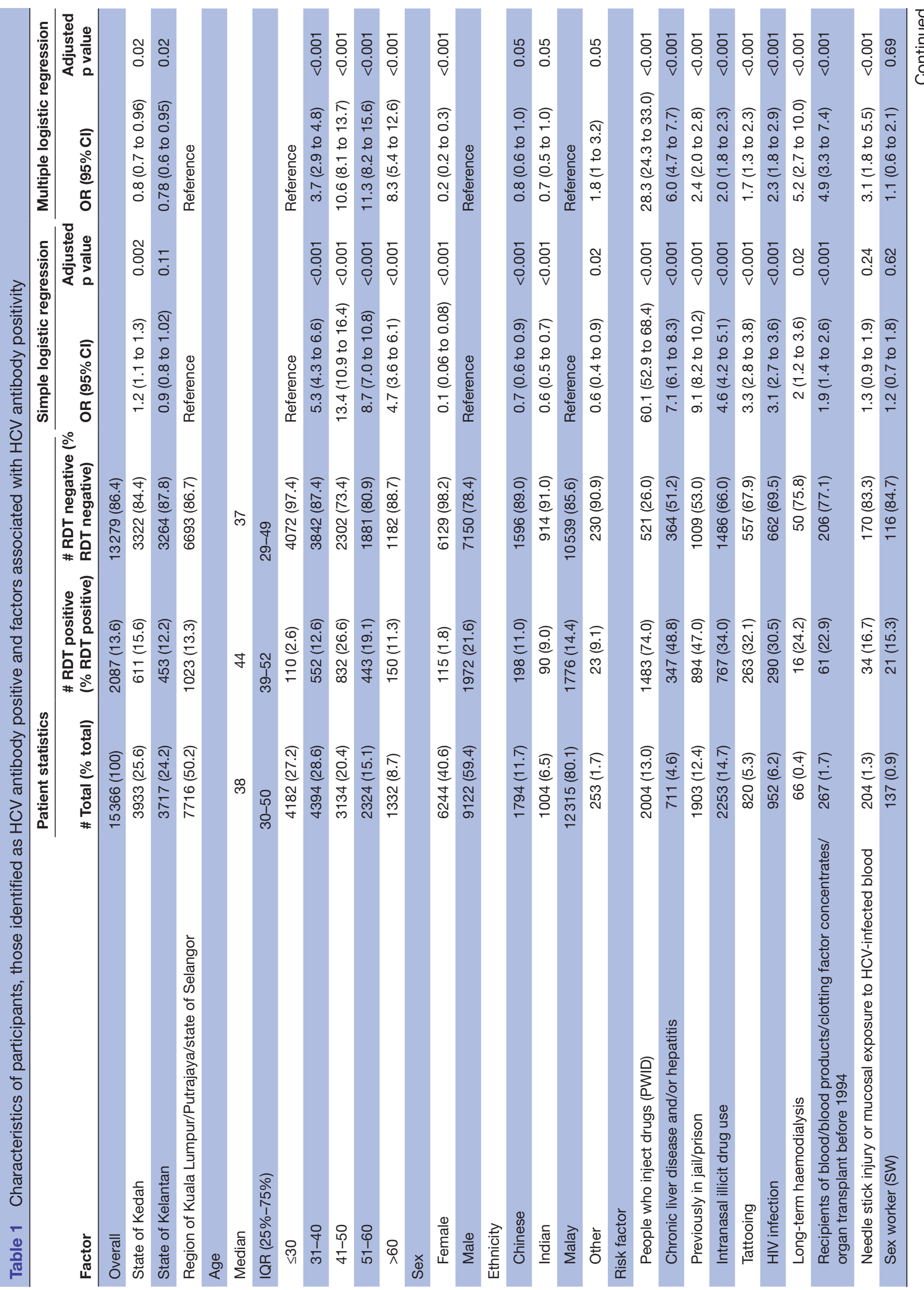


During the study period, 67/632 (10.6\%) participants were still undergoing treatment, of which 11/67 (16.4\%) were non-cirrhotic and were 56/67 (83.6\%) cirrhotic. No patient reported discontinuing treatment due to serious treatment-related adverse events or death.

Among those who returned for SVR testing, there were $7 / 209$ (3.3\%) participants who experienced treatment failure, all of whom were non-cirrhotic.

\section{Cascade outcomes among key populations}

Of the enrolled participants, 1944/15 299 (12.7\%) were PWID, of whom $1464 / 1944(75.3 \%)$ were HCV seropositive, compared with 556/13 $355(4.2 \%)$ in the nonPWID population $(\mathrm{p}<0.001)$ (online supplemental table 2 ). There was no significant difference in the uptake of HCV confirmatory RNA testing according to PWID status. However, slightly lower proportions of PWID were RNA positive compared with the non-PWID group: 877/1063 $(82.5 \%)$ and $364 / 418(87.1 \%)$, respectively $(\mathrm{p}=0.04)$. Similar proportions of RNA-positive PWID completed pretreatment assessment, initiated treatment, completed treatment, received SVR testing and achieved SVR compared with non-PWID. There was also no difference in the outcomes between PWID and non-PWID when stratified by treatment site (PHC vs hospital).

There were 983/15 299 (6.4\%) participants who were PLHIV and, of these, 298/983 (30.3\%) were HCV seropositive compared with $1722 / 14316(12.0 \%)$ in the non-PLHIV group $(\mathrm{p}<0.001)$. There was no significant difference in the uptake of positive HCV confirmatory RNA testing according to HIV status. Slightly lower proportions of RNA-positive PLHIV completed pretreatment assessment compared with the non-PLHIV group: $145 / 197(73.6-\%)$ and 846/1250 (81.0\%), respectively $(\mathrm{p}=0.02)$. Similar proportions of RNA-positive PLHIV initiated treatment, completed treatment, received SVR testing and achieved SVR compared with non-PLHIV. There was also no difference in the outcomes between PLHIV and non-PLHIV when stratified by treatment site (PHC vs hospital).

\section{Turnaround time between HCV care cascade steps}

The total median time (IQR) in days between of $\mathrm{HCV}$ serological testing and treatment initiation was 214 (148269) (table 2). The turnaround time from completion of pretreatment assessment to treatment initiation was longest at 103 (47-149) days, followed by time from RNA results returned to patient to completion of pretreatment assessment (33 (13-58) days). There were some differences in the times from HCV serological testing to treatment initiation between those referred for treatment at PHCs and at hospitals: 217 (167-277) days versus 191 (124-249) days, respectively $(\mathrm{p}=0.004)$. In addition, there was no difference in the turnaround times along the HCV care cascade between PLHIV and non-PLHIV; and the only significant difference in time from HCV serological testing to treatment initiation between PWID and 


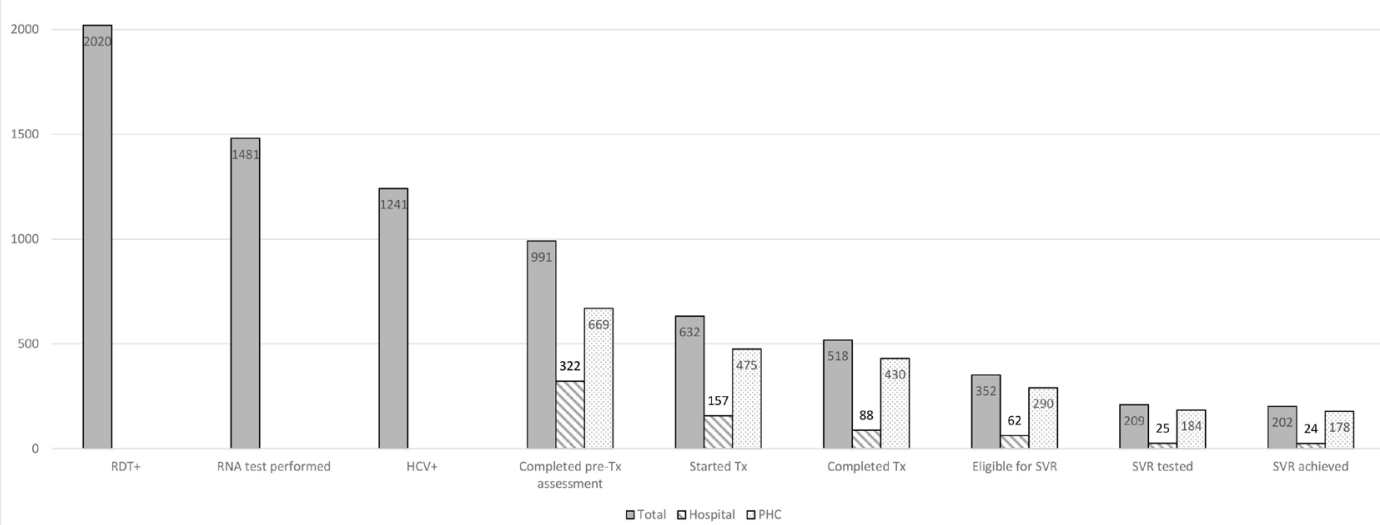

\begin{tabular}{|c|c|c|c|c|c|c|c|c|c|c|}
\hline \multirow{2}{*}{$\begin{array}{c}\text { HCV care } \\
\text { cascade step }\end{array}$} & \multirow[t]{2}{*}{ Overall } & \multicolumn{3}{|c|}{ Tx site: PHC } & \multicolumn{3}{|c|}{ Tx site: Hospital } & \multirow{2}{*}{$\begin{array}{l}\text { Adjusted p- } \\
\text { values for } \\
\text { PHC overall } \\
\text { vs Hospital } \\
\text { overall }\end{array}$} & \multirow{2}{*}{$\begin{array}{l}\text { Adjusted p- } \\
\text { values for } \\
\text { PHC cirrhotic } \\
\text { vs Hospital } \\
\text { cirrhotic }\end{array}$} & \multirow{2}{*}{$\begin{array}{c}\text { Adjusted p- } \\
\text { values for } \\
\text { PHC non- } \\
\text { cirrhotic vs } \\
\text { Hospital non- } \\
\text { cirrhotic } \\
\end{array}$} \\
\hline & & \multirow{4}{*}{ Overall } & \multirow{4}{*}{ Cirrhotic } & \multirow{4}{*}{ Non-cirrhotic } & \multirow{4}{*}{ Overall } & \multirow{4}{*}{ Cirrhotic } & \multirow{4}{*}{ Non-cirrhotic } & & & \\
\hline RDT+ & $\begin{array}{r}2020 / 15299 \\
(13.2 \%)\end{array}$ & & & & & & & & & \\
\hline RNA test done & $\begin{array}{r}1481 / 2020 \\
(73.3 \%) \\
\end{array}$ & & & & & & & & & \\
\hline RNAt & $\begin{array}{r}1241 / 1481 \\
(83.8 \%) \\
\end{array}$ & & & & & & & & & \\
\hline $\begin{array}{l}\text { Completed pre-Tx } \\
\text { assessments }\end{array}$ & $991 / 1241(79.9 \%)$ & $669 / 991(67.5 \%)$ & $73 / 669(10.9 \%)$ & $596 / 669(89.1 \%)$ & $322 / 991(32.5 \%)$ & $258 / 322(80.1 \%)$ & $64 / 322(19.9 \%)$ & & & \\
\hline Started $T x$ & $632 / 475(63.8 \%)$ & $475 / 669(71.0 \%)$ & $59 / 73(80.8 \%)$ & $416 / 596(69.6 \%)$ & $157 / 322(48.8 \%)$ & $121 / 258(46.9 \%)$ & $36 / 64(56.3 \%)$ & $<0.001$ & $<0.001$ & 0.19 \\
\hline Completed Tx & $518 / 632(82.0 \%)$ & $430 / 475(90.5 \%)$ & $39 / 59$ (66.1\%) & $391 / 416(94.0 \%)$ & $88 / 157(56.1 \%)$ & $63 / 121(52.1 \%)$ & $25 / 36(69.4 \%)$ & $<0.001$ & 0.52 & $<0.001$ \\
\hline Eligible for SVR & $352 / 518(68.0 \%)$ & $290 / 430(67.4 \%)$ & $20 / 39(51.3 \%)$ & $270 / 391(69.1 \%)$ & $62 / 88(70.5 \%)$ & $39 / 63(61.9 \%)$ & $23 / 25(92.0 \%)$ & 1.00 & 1.00 & 0.14 \\
\hline SVR tested & $209 / 352$ (59.4\%) & $184 / 290$ (63.4\%) & $8 / 20(40.0 \%)$ & $176 / 270(65.2 \%)$ & $25 / 62(40.3 \%)$ & $12 / 39(30.8 \%)$ & $13 / 23(56.5 \%)$ & $<0.001$ & 1.00 & 1.00 \\
\hline SVR achieved & $202 / 209$ (96.7\%) & $178 / 184(96.7 \%)$ & $8 / 8(100 \%)$ & $170 / 176(96.6 \%)$ & $24 / 25(96.0 \%)$ & $12 / 12(100 \%)$ & $12 / 13(92.3 \%)$ & 1.00 & 1.00 & 1.00 \\
\hline
\end{tabular}

Figure 2 Retention of patients in the HCV care cascade by treatment site and cirrhotic status. PHC, primary healthcare clinic; RDT, rapid diagnostic test; SVR, sustained virological response; Tx, treatment.

non-PWID was 220 (164-279) days versus 197 (133-248) days, respectively $(\mathrm{p}=0.02)$ (online supplemental table 3$)$.

\section{Cost of HCV testing}

In a cohort of 10000 patients with an RDT-positive rate of $13.6 \%$ and viraemic rate among antibody positive participants of $84.5 \%$, the total cost for testing per treated patient was US\$136.21. Over a 30-year time period, the overall cost per 10000 patients and quality-adjusted life-years (QALYs) were US\$6 921839 and US\$172 036, compared with the cost and QALYs of no action of US $\$ 11$ 725196 and US\$170 774. Compared with no testing, a simplified decentralised HCV testing and treatment model in primary healthcare settings could result in cost savings of US $\$ 4.8$ million per 10000 persons tested over the span of 30 years. The disease burden avoided for decompensated cirrhosis, hepatocellular carcinoma and liver disease-related deaths, compared with the burden with no action, were 116, 68 and 117 cases, respectively (online supplemental table 4).

\section{DISCUSSION}

Overall, this project demonstrated the effectiveness and feasibility of a simplified, decentralised HCV testing and treatment model in primary care settings that targeted high-risk groups in Malaysia. Good outcomes were attained across most steps of the cascade of care when patients were offered treatment at decentralised sites compared with centralised hospitals, however, considerable attrition was reported for linkage to care.
A distinctive feature of this model was its demonstration that HCV case-finding at PHCs using RDTs is both feasible (reflected by the marked increase in testing uptake, with more than 15000 individuals being tested in 10 months) and achieved a high rate of case-finding (prevalence $13.2 \%$ ). This was achieved through a targeted HCV casefinding strategy of identifying high-risk patients within the PHC catchment population. The resultant enrolled population included high proportions of key populations including PWID, PLHIV, MSM, SW, intranasal illicit drug users, those who were previously in prisons as well as individuals with chronic liver disease or a history of invasive medical procedures. Targeting these populations resulted in an overall high yield of HCV-positive cases (13.2\%).

While overall retention was good for most steps in the cascade, our findings also highlight some cascade steps where there was significant attrition and suboptimal linkage following a positive HCV antibody RDT that provide opportunities for improvement. There were 539 patients $(26.7 \%)$ who did not have a confirmatory viral load test and a further 250 (20.1\%) who had an HCV viral load test that was positive but did not receive pretreatment assessments (ie, a total of $789(39.1 \%)$ of the HCV antibody-positive individuals). In addition, there was attrition of RNA positive patients initiating treatment, but markedly more so in patients that received treatment at hospitals compared with those treated at PHC (48.8\% vs $71.0 \%, \mathrm{p}<0.001)$.

It is likely that at both points in the cascade a key driver of attrition is the provision of services at hospitals. All 


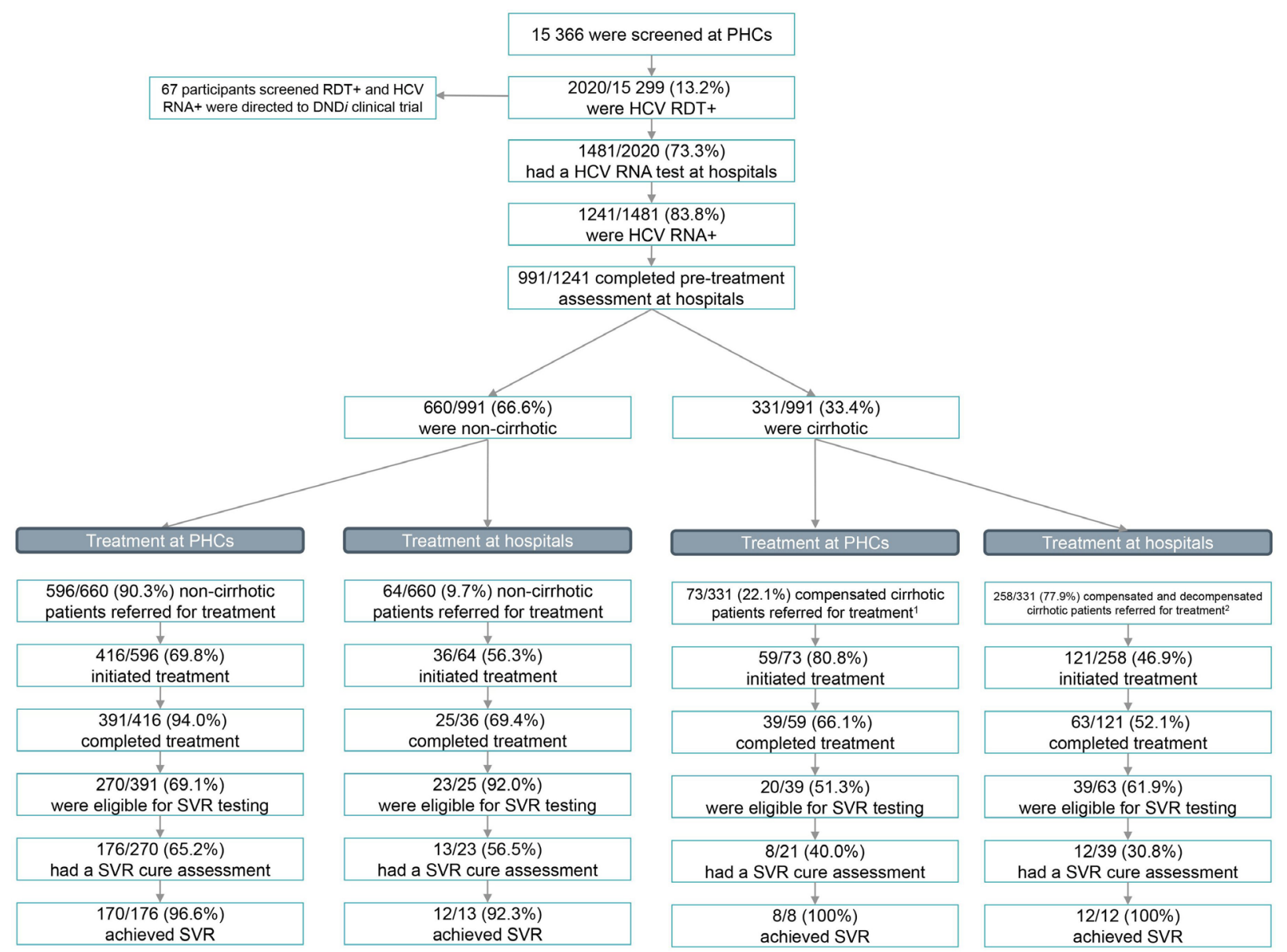

Figure 3 Flowchart of treatment sites for cirrhotic versus non-cirrhotic patients. ${ }^{1}$ These compensated cirrhotic patients were referred for treatment (sofosbuvir/daclatasavir without ribavirin) at six PHCs in the state of Kedah. ${ }^{2}$ These compensated and decompensated cirrhotic patients were referred for treatment at hospitals. PHC, primary healthcare clinic; RDT, rapid diagnostic test; SVR, sustained virological response; Tx, treatment.

seropositive participants were referred up from PHCs to designated hospital sites for viral load testing and subsequent pretreatment assessment, rather than having on-site blood sample collection for HCV viral load and pretreatment assessment. This was due to the preenrolment requirements of the DND $i$ clinical trial (viral load and pretreatment assessment). During follow-up calls, study staff logged the following reasons for attrition from hospital visits; the distance participants had to travel to the hospital (online supplemental table 1); high transportation costs. In addition, it is likely that follow-up of patients was not carried out to the highest standard due to limited information exchange between hospital and PHC staff and poor tracking and tracing of patients lost. In addition, limited appointment availability and long lead-times for blood collection at the hospitals; and a reluctance to attend hospital appointments because of fear of stigmatisation in contrast to the 'high-risk population-friendly' PHCs were also likely causes of attrition of patients throughout the cascade.

These findings are consistent with evidence from a recent systematic review which reported lower rates of linkage to care and treatment uptake in partially decentralised models of care (29 studies) compared with fully decentralised models of care (ie, all testing and treatment provided at a single site). Although, this finding was only reported for key populations whereas results in the general population were heterogeneous. In addition, only $49 \%$ of studies included in this review were from low and middleincome countries. ${ }^{25}$ Other studies with similar models of partially decentralised HCV care assessed within existing public health systems have reported poorer retention between diagnosis of HCV and treatment $59.3 \%$ in the Cherokee nation HCV elimination programme and 73.7\% for partially decentralised arm in the HEAD-Start Project Delhi. ${ }^{26}{ }^{27}$ Whereas, high rates of retention have been reported in several studies where fully decentralised care is provided. ${ }^{28} 29$

Preliminary analyses of loss to follow-up led to changes in the national programme to allow for the provision of HCV treatment at PHCs for non-cirrhotic patients (including patients who were coinfected with HIV), from quarter 3 of 2019. This change enabled a subanalysis of patients, according to where their treatment was provided. We observed a significant improvement cascade of care retention when patients had treatment provided at a PHC compared with receiving treatment at a hospital. In addition, at six PHCs (in the state of Kedah), treatment of 


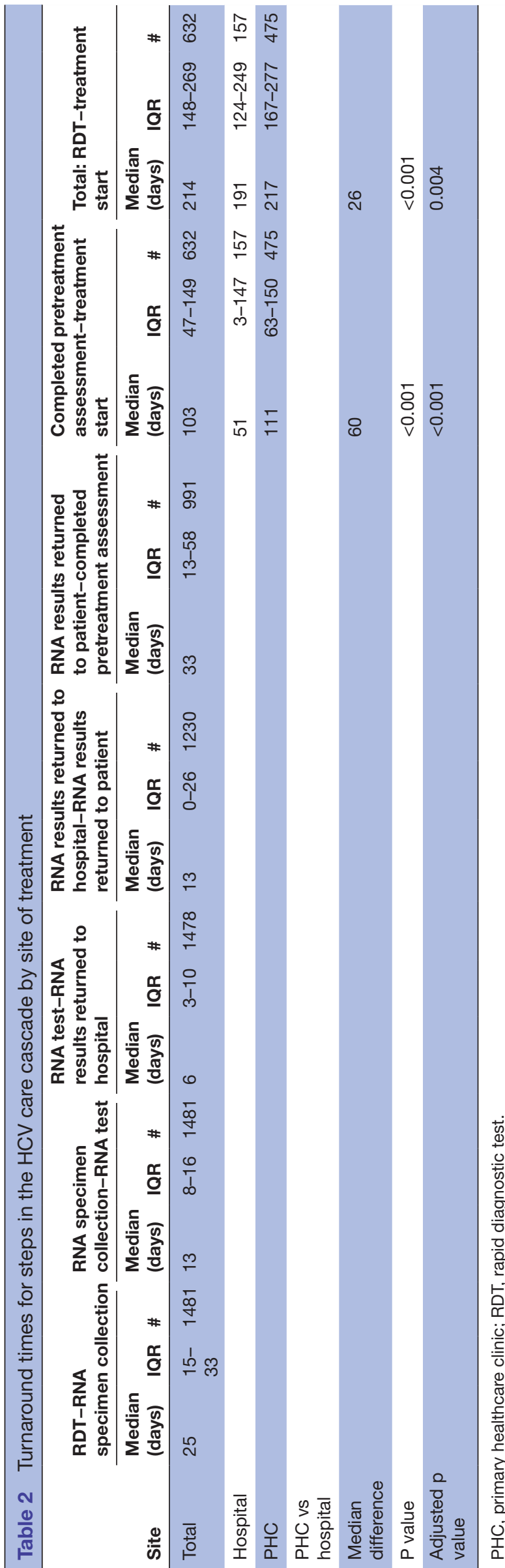

a small cohort of compensated cirrhotic patients $(n=59$, using sofosbuvir/daclatasavir without ribavirin) ${ }^{18} 19$ was successful.

There were several key limitations in this study: first, the study is observational in nature, with inherent challenges taking into account the many confounders affecting outcomes across the cascade of care, particularly when comparing PHCs versus hospitals; second, standard of care HCV practices evolved during the conduct of the study-decentralisation of HCV treatment under the $\mathrm{MOH}$ national programme for non-cirrhotic patients to PHCs, including patients who were coinfected with HIV and a small cohort of compensated cirrhotic patients, commenced in quarter 3 of 2019; third, the costing estimates did not take into account the differential costs of the HCV care pathway between PHCs and hospitals as well as the costs to the patients.

The major challenges encountered were due to the COVID-19 pandemic, which resulted in delays to treatment initiation (65 (24-112) days pre-COVID-19 vs 154 $(127-211)$ days post-COVID-19, $\mathrm{p}<0.001)$. These delays may have contributed to increased numbers of patients lost to follow-up. In particular, loss to follow-up in treatment initiation, follow-up treatment visits and SVR testing during the COVID-19 outbreak may have been higher among key populations, such as PWID, who were reportedly less willing to travel to treatment sites amid increased police surveillance during COVID-19 lockdowns due to fear of arrest. Indeed, others have also reported access to all healthcare and other services for PWID was affected by COVID-19. ${ }^{30}$ In addition, HCV screening at PHCs was reduced, laboratory turnaround times were increased and an increase in SVR samples being misplaced was reported during the COVID-19 outbreak, due to prioritisation of laboratory resources for processing COVID-19 samples.

Initiatives by the MOH after the study started have led to a tremendous expansion in screening and treatment for HCV. ${ }^{31-34}$ The evidence from this study has catalysed plans for $\mathrm{MOH}$ to roll-out decentralised HCV care from the 25 sites to all PHCs nationwide, in a stepwise manner from quarter 1 of $2020 .^{34}$ The model for scale-up builds on key aspects that were integral to this study design, including the use of RDTs for point of care results, optimal turnaround times and the use of DAA therapies at PHCs for non-cirrhotic and uncomplicated cases of HCV, where there is capacity present on-site. In addition, $\mathrm{MOH}$ has already recently further decentralised services by ensuring venous blood collection for HCV viral load confirmation and reflex biochemistry blood tests for pretreatment assessments, including AST to Platelet Ratio Index scores, will be carried out at PHCs rather than referring these patients to hospitals. ${ }^{18}$ This includes sending the sample for either HCVCAg or HCV RNA testing at a designated hospital or laboratory or use of on-site GeneXpert testing making the model a fully decentralised care package.

In addition to the scale-up of this fully decentralised model of HCV care at PHCs nationally, this study has provided evidence, with regards to the yield of 
HCV RDT+, for the MOH to continue the successful approach of targeting high-risk groups of individuals for HCV screening. To this end, the $\mathrm{MOH}$ has also started programmes in prisons ${ }^{35}$ and drug rehabilitation centres and is developing plans for novel screening strategies, including self-testing, to further target key populations including MSM, transgender people and SW. To ensure successful HCV programmes can be implemented within these populations, the $\mathrm{MOH}$ has begun to drive the coordination of different government departments including primary care, public health, the Ministry of Home Affairs and relevant non-governmental organisations (NGOs).

Further lessons from this study that are being adopted for scale-up include an emphasis on the importance of developing robust monitoring and evaluation and data collection systems at every step of the HCV care cascade. The use of a central database for capturing data in a systematic way during this study can be translated for use in the national programme in simplified and practical ways. As well as the importance of strategies to raise community awareness, outreach activities and engagement with NGOs.

\section{CONCLUSIONS}

In conclusion, using an innovative model of partially decentralised care, this study demonstrated a high rate of case-finding for HCV-positive individuals. There were significantly higher levels of retention in the care cascade when patients were treated at PHCs compared with hospitals supporting existing evidence of improved outcomes using decentralised care. Several improvements were made during the study and in the national programme to address these limitations, including the decentralisation of confirmatory testing and pretreatment assessment and the provision of HCV treatment at PHCs. This optimised model of fully decentralised HCV care, is now being adopted by $\mathrm{MOH}$ as part of a scale-up nationwide and serves as a good model for implementation in other settings.

\section{Author affiliations}

${ }^{1}$ FIND, Geneva, Switzerland

${ }^{2}$ Clinical Research Centre, Hospital Sultanah Bahiyah, Alor Setar, Malaysia

${ }^{3}$ Hospital Ampang, Ampang Jaya, Malaysia

${ }^{4}$ Drugs for Neglected Diseases initiative, Geneva, Switzerland

${ }^{5}$ Hospital Sultanah Bahiyah, Alor Setar, Malaysia

${ }^{6}$ Hospital Raja Perempuan Zainab II, Kota Bharu, Malaysia

${ }^{7}$ Hospital Selayang, Batu Caves, Malaysia

${ }^{8}$ Division of Clinical Tropical Medicine, Center of Infectious Diseases, University Hospital Heidelberg, Heidelberg, Germany

${ }^{9}$ Division of Genetics, Department of Medicine, Brigham and Women's Hospital, Harvard Medical School, Boston, Massachusetts, USA

${ }^{10}$ Belozersky Institute of Physico-Chemical Biology, Moscow State University,

Moscow, Russia

${ }^{11}$ Institute for Technology Assessment, Massachusetts General Hospital, Harvard

Medical School, Boston, Massachusetts, USA

${ }^{12}$ Hospital Sungai Buloh, Sungai Buloh, Malaysia

${ }^{13}$ Institute for Medical Research, Kuala Lumpur, Malaysia

${ }^{14}$ Disease Control Division, Ministry of Health, Putrajaya, Malaysia

${ }^{15}$ Family Health Development Division, Ministry of Health, Putrajaya, Malaysia
${ }^{16}$ Global HIV, Hepatitis \& STI Programmes, World Health Organization, Geneva, Switzerland

Acknowledgements The authors would like to thank Unitaid for the support and funding; study participants for their involvement, and partners and colleagues for the support and implementation of this study - study teams and their colleagues from the 25 PHCs, 5 hospitals and IMR, DNDi, Clinical Research Malaysia (CRM) and Clinical Research Centre; in particular: Dr Akhmal Yusof and the study coordinators from CRM; Family Medicine Specialists from the 25 PHCs - Dr Idora Ibrahim, Dr Zamri Mansor, Dr Siti Aishah Johari, Dr Habshoh Hat, Dr Fazlin Suhana Othman, Dr Jamilah Abdullah, Dr Norzaihan Hassan, Dr Tuan Zailina Tuan Ngah, Dr Rahiza Ab Rahman, Dr Nik Harlina Roza Nik Kazim, Dr Roshana Mohamed Yasin, Dr Norashidah Abdullah, Dr Mohd Sukarno Saud, Dr Narul Aida Salleh. Dr Siti Rohani Mohamed Alias, Dr Noor Harzana Harrun, Dr Husni Hussain, Dr Rafidah Mohd Rafie, Dr Radziah Jabir, Dr Nazhatussima Suhaili, Dr Sheela Bai a/p Pannir Selvam, Dr Wan Noor Azlin Wan Idris, Dr Zienna Zufida Zainol Rashid, Dr Ziti Akthar Supian, Dr Fauziah Ahmad and Dr Vickneswari a/p Ayadurai; the clinical trial assistants, site supervisor and clinical research assistants from DNDi: Wan Nur Illyana Wan Yusoff, Noor Annisa Darman, Muhammad Adib Abd Wahab, Matron Wakia Wahab, Nurul Amalina Md Zemi, Nur Farahana Zakaria, Nur Aliya Ahmat @ Ahmad, Nurul Fazira Basran, Muhammad Hafiz Idris, Putri Shafinaz Sharudin, Muhammad Taufik Nasaruddin and Khairul Fidaa Khairul Bazli; colleagues from DNDi: François Bompart, Molly Jagpal, Han Yang Chung, Muhammad Hasif Mahmad Fauzi, Kamsiah Hasbullah, Azeera Alvina Aben, Tilakbabu Ramanaidu, Nyap Ming Tim, Francois Simon, Tania Bilyk and Guillaume Drapeau; and present and past colleagues from FIND: Catharina Boehme, Bill Rodriguez, Zachary Katz, Francesco Marinucci, Violet Chihota, Natalie Jotikasthira, Cristina Dang, Jeremy OBrien, Flavio Ambrogiani and Dominique Rabian. The authors would also like to thank the following civil society organisations and governmental agency for their support and contribution - Persatuan Cahaya Harapan Negeri Kedah/Perlis, Persatuan Perantaraan Pesakit Kelantan, Persatuan Insaf Murni Malaysia, the Malaysian AIDS Council and the National Anti-Drug Agency; and the Director-General of Health, Malaysia, for his support throughout the conduct of this study and permission to publish the findings. Editorial support, under the direction of the authors and funded by FIND, was provided by Adam Bodley.

Contributors JM designed data collection tools, implemented the study, monitored data collection for the study, cleaned and analysed the data, and drafted and revised the paper. She is guarantor. SShilton initiated the collaborative project, designed the study and data collection tools, implemented the study, monitored data collection for the study and drafted and revised the paper. She is guarantor. XHS designed data collection tools, implemented the study, monitored data collection for the study, cleaned and analysed the data, and revised the draft paper. CHK revised the draft paper. RMS, ZZ, NAB, HO, SK and RH implemented the study and monitored data collection for the study. SSiva designed the study and data collection tools, implemented the study, monitored data collection for the study and revised the draft paper. RJR implemented the study and monitored data collection for the study. MG and AT wrote the statistical analysis plan, cleaned and analysed the data, and revised the draft paper. MA and JC analysed the data and revised the draft paper. J-MP initiated the collaborative project, implemented the study, and revised the draft paper. RMZ initiated the collaborative project, designed and implemented the study, and monitored data collection for the study. CM implemented the study, monitored data collection for the study, and revised the draft paper. FY, NHN, FI and RZ implemented the study. IA-M initiated the collaborative project, designed and implemented the study, and revised the draft paper. SM initiated the collaborative project and implemented the study. PE drafted and revised the paper. MRAH initiated the collaborative project, designed and implemented the study, monitored data collection for the study and revised the draft paper. All authors revised the paper critically for intellectual content and approved the final version.

Funding This study was funded by Unitaid as part of HEAD-Start (Hepatitis Elimination through Access to Diagnostics).

Map disclaimer The inclusion of any map (including the depiction of any boundaries therein), or of any geographic or locational reference, does not imply the expression of any opinion whatsoever on the part of BMJ concerning the legal status of any country, territory, jurisdiction or area or of its authorities. Any such expression remains solely that of the relevant source and is not endorsed by BMJ. Maps are provided without any warranty of any kind, either express or implied.

Competing interests None declared.

Patient consent for publication Not applicable. 
Ethics approval This study involves human participants and the ethics committee that approved this study is the Malaysian Medical Research and Ethics Committee (approval number: NMRR-18-2282-43132). Participants gave informed consent to participate in the study before taking part.

Provenance and peer review Not commissioned; externally peer reviewed.

Data availability statement Data are available upon reasonable request.

Supplemental material This content has been supplied by the author(s). It has not been vetted by BMJ Publishing Group Limited (BMJ) and may not have been peer-reviewed. Any opinions or recommendations discussed are solely those of the author(s) and are not endorsed by BMJ. BMJ disclaims all liability and responsibility arising from any reliance placed on the content. Where the content includes any translated material, BMJ does not warrant the accuracy and reliability of the translations (including but not limited to local regulations, clinical guidelines, terminology, drug names and drug dosages), and is not responsible for any error and/or omissions arising from translation and adaptation or otherwise.

Open access This is an open access article distributed in accordance with the Creative Commons Attribution Non Commercial (CC BY-NC 4.0) license, which permits others to distribute, remix, adapt, build upon this work non-commercially, and license their derivative works on different terms, provided the original work is properly cited, appropriate credit is given, any changes made indicated, and the use is non-commercial. See: http://creativecommons.org/licenses/by-nc/4.0/.

\section{ORCID iDs}

Sonjelle Shilton http://orcid.org/0000-0003-1569-8758

Jagpreet Chhatwal http://orcid.org/0000-0001-8741-4430

\section{REFERENCES}

1 Mohd Hanafiah K, Groeger J, Flaxman AD, et al. Global epidemiology of hepatitis $C$ virus infection: new estimates of age-specific antibody to HCV seroprevalence. Hepatology 2013;57:1333-42.

2 World Health Organization. Guidelines on hepatitis B and C testing, 2017. Available: http://www.who.int/hepatitis/publications/guidelineshepatitis-c-b-testing/en/

3 World Health Organization. Global progress report on HIV, viral hepatitis and sexually transmitted infections, 2021, 2021. Available: https://www.who.int/publications/i/item/9789240027077

4 World Health Organization. Global health sector strategy on hepatitis 2016-2021, 2016. Available: https://apps.who.int/iris/bitstream/ handle/10665/246177/WHO-HIV-2016.06-eng.pdf

5 World Health Organization. Guidelines for the care and treatment of persons diagnosed with chronic hepatitis $C$ virus infection, 2018. Available: https://apps.who.int/iris/bitstream/handle/10665/273174/ 9789241550345-eng.pdf?ua=1

6 Muhamad NA, Ab Ghani RM, Abdul Mutalip MH, et al. Seroprevalence of hepatitis $B$ virus and hepatitis $C$ virus infection among Malaysian population. Sci Rep 2020;10:21009.

7 Ministry of Health Malaysia. Integrated biological and behavioural surveillance Putrajaya, 2014. Available: http://www.moh.gov.my/moh/ resources/Penerbitan/Laporan/Umum/Final_Report_IBBS_2014_ DGendorsed.pdf

8 Ministry of Health Malaysia. Global AIDS monitoring 2020 - Malaysia HIV/AIDS progress report, 2020. Available: https://www.moh.gov.my/ moh/resources/Penerbitan/Laporan/Umum/Laporan_Global_AIDS Monitoring_2020_new.pdf

9 Ministry of Health Malaysia. Size estimation of key populations in Malaysia Putrajaya, 2018. Available: https://www.moh.gov.my/index. $\mathrm{php} / \mathrm{dl} / 554756755 \mathrm{a} 584 \mathrm{a} 69615852686269394 \mathrm{~d} 59584276636 \mathrm{~d} 4675$ 4c3156746457307655464e4658326c7558303168624746356332 6c68587a49774d5467756347526d

10 Ministry of Health Malaysia. National strategic plan for hepatitis B and C 2019-2023, 2019. Available: https://www.moh.gov.my/moh/ resources/Penerbitan/Pelan\%20Strategik\%20/NSP_Hep_BC_2019_ 2023.pdf

11 Rahman F. At the edge of a miracle: the hepatitis $\mathrm{C}$ virus (HCV) epidemic in Malaysia Kuala Lumpur, 2017. Available: https://www. mac.org.my/v3/at-the-edge-of-a-miracle/

12 Hiebert L, Hecht R, Soe-Lin S, et al. A stepwise approach to a national hepatitis $C$ screening strategy in Malaysia to meet the who 2030 targets: proposed strategy, coverage, and costs. Value Health Reg Issues 2019;18:112-20.
13 Ministry of Health Malaysia. Annual report, 2019. Available: https:// myhdw.moh.gov.my/public/documents/20186/150089/Annual+ Report+MoH+2019/24433263-99fc-4892-bd2e-6deb2e9fcb26

14 Lim T, Sivasampu S, Nadia F. Chapter 1: Overview on primary care services in Malaysia. National healthcare establishments \& workforce statistics 2008-2009, 2010.

15 Andrieux-Meyer I, Tan S-S, Thanprasertsuk S, et al. Efficacy and safety of ravidasvir plus sofosbuvir in patients with chronic hepatitis $\mathrm{C}$ infection without cirrhosis or with compensated cirrhosis (STORMC-1): interim analysis of a two-stage, open-label, multicentre, single arm, phase 2/3 trial. Lancet Gastroenterol Hepatol 2021;6:448-58.

16 Clinical trial protocol: open label phase II/III, multicenter, trial to assess the efficacy, safety, tolerance, and pharmacokinetics of sofosbuvir plus ravidasvir in HCV (+/- HIV) chronically infected adults with no or compensated cirrhosis in Thailand and Malaysia: drugs for neglected diseases initiative, 2018. Available: https://dndi.org/wpcontent/uploads/2020/06/DNDi-Clinical-Trial-Protocol-SOF-RDV-V8. pdf [Accessed Aug 2020].

17 Ministry of Health Malaysia. Hepatitis C screening, testing and treatment guidelines, 2017.

18 Ministry of Health Malaysia. Management of chronic hepatitis $C$ in adults, 2019.

19 Pol S, Corouge M, Vallet-Pichard A. Daclatasvir-sofosbuvir combination therapy with or without ribavirin for hepatitis $\mathrm{C}$ virus infection: from the clinical trials to real life. Hepat Med 2016;8:21-6.

20 Armstrong RA. When to use the Bonferroni correction. Ophthalmic Physiol Opt 2014;34:502-8.

21 Chhatwal J, Chen Q, Bethea ED, et al. Hep C calculator: an online tool for cost-effectiveness analysis of DAAs. Lancet Gastroenterol Hepatol 2018;3:819.

22 Chhatwal J, Ferrante SA, Brass C, et al. Cost-effectiveness of boceprevir in patients previously treated for chronic hepatitis C genotype 1 infection in the United States. Value Health 2013;16:973-86.

23 Chhatwal J, Kanwal F, Roberts MS, et al. Cost-effectiveness and budget impact of hepatitis $C$ virus treatment with sofosbuvir and ledipasvir in the United States. Ann Intern Med 2015;162:397-406.

24 World Health Organization. Meeting report: viral hepatitis strategic information and modelling reference group Geneva, 2016. Available: https://www.who.int/hepatitis/publications/strategic-informationmodelling-meeting/en/

25 Oru E, Trickey A, Shirali R, et al. Decentralisation, integration, and task-shifting in hepatitis $C$ virus infection testing and treatment: a global systematic review and meta-analysis. Lancet Glob Health 2021;9:e431-45.

26 Markby J, Gupta E, Soni D. Feasibility, effectiveness and cost of a decentralized HCV care model among the general population in Delhi, India. Liver Int 2021 doi:10.1111/liv.15112

27 Mera J, Williams MB, Essex W, et al. Evaluation of the Cherokee nation hepatitis $C$ virus elimination program in the first 22 months of implementation. JAMA Netw Open 2020;3:e2030427.

28 Draper BL, Htay H, Pedrana A, et al. Outcomes of the CT2 study: a 'one-stop-shop' for community-based hepatitis $\mathrm{C}$ testing and treatment in Yangon, Myanmar. Liver Int 2021;41:2578-89.

29 Zhang M, O'Keefe D, Craig J, et al. Decentralised hepatitis C testing and treatment in rural Cambodia: evaluation of a simplified service model integrated in an existing public health system. Lancet Gastroenterol Hepatol 2021;6:371-80.

30 Grebely J, Cerdá M, Rhodes T. COVID-19 and the health of people who use drugs: what is and what could be? Int J Drug Policy 2020;83:102958.

31 Chan H-K, Hassali MA, Md Said R, et al. A two-year outcome evaluation of government-led initiative to upscale hospital-based hepatitis $\mathrm{C}$ treatment using a standard two-drug regimen in Malaysia Hepat Mon 2021;21:e113226.

32 Md Said R, Mohd Zain R, Chan H-K, et al. Find the missing millions: Malaysia's experience with nationwide hepatitis $\mathrm{C}$ screening campaign in the general population. J Viral Hepat 2020;27:638-43.

33 Hassan MRA, Chan H-K. Comment on: Projections of the healthcare costs and disease burden due to hepatitis $\mathrm{C}$ infection under different treatment policies in Malaysia, 2018-2040. Appl Health Econ Health Policy 2020;18:139-40.

34 Chan H-K, Hassali MA, Md Said R, et al. Treatment coverage and drug expenditure in hepatitis C patients from 2013 to 2019: a journey of improving treatment accessibility in Malaysia through Government-led initiatives. Hepat Mon 2020;20:e107372.

$35 \mathrm{He} \mathrm{T}$, Li K, Roberts MS, et al. Prevention of hepatitis C by screening and treatment in U.S. prisons. Ann Intern Med 2016;164:84-92. 